\title{
Social dysfunction in schizophrenia: An investigation of the GAF scale's sensitivity to deficits in social cognition
}

\author{
Deirdre A. Robertson, April Hargreaves, Eric B. Kelleher, Derek Morris, Michael Gill, \\ Aiden Corvin, Gary Donohoe*
}

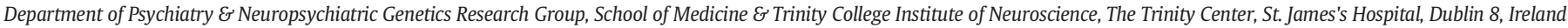

\section{A R T I C L E I N F O}

\section{Article history:}

Received 19 September 2012

Received in revised form 3 January 2013

Accepted 10 January 2013

Available online $\mathrm{xxxx}$

\section{Keywords:}

Schizophrenia

Social cognition

Neurocognition

Global assessment of functioning (GAF) scale

Psychosocial function

\begin{abstract}
A B S T R A C T
Background: Schizophrenia is accompanied by significant impairment in psychosocial functioning, which is only partially explained by clinical symptom severity. Recently, these impairments have been strongly associated with deficits in neurocognition and social cognition. Although the Global Assessment of Function (GAF) scale remains the most widely used measure of psychosocial function in clinical practice, it is unclear whether this instrument is sensitive to changes in cognition, or merely provides a snapshot of symptom severity. To investigate this, we assessed whether variation in GAF score was explained by performance on measures of neurocognitive and social cognition, particularly after variation associated with symptom severity had been accounted for.

Methods: 216 patients with schizophrenia were assessed using the GAF scale, two theory of mind tasks (the 'Hinting' task and 'Reading the Eyes in the Mind' task), and a neuropsychological battery sensitive to the areas of deficit typically seen in schizophrenia - IQ episodic memory, working memory and attentional control.

Results: Using linear regression analysis, symptom severity explained $24 \%$ of the variance in GAF scores $(F(3,188)=21.14, p<.001)$. While neuropsychological performance explained a further $4.7 \%$ of variation $\left(r_{\text {change }}^{2}=.047, F_{\text {change }}(1,187)=12.63, p<.001\right)$, social cognition did not explain any further variance in functioning $\left(r_{\text {change }}^{2}=.006, F_{\text {change }}(1,186)=1.63, p=.20\right)$.

Conclusion: These data indicate that GAF scores are primarily sensitive to variation in clinical symptoms severity and not at all sensitive to variation in social cognition, an important determinant of real world outcome. Doing so highlights the need to supplement the measurement of psychosocial function using the GAF in clinical practice with functional measures that are more sensitive to deficits in social cognition.
\end{abstract}

(c) 2013 Published by Elsevier B.V.

\section{Introduction}

Although antipsychotic medications help manage clinical symptom severity, many people with schizophrenia remain unemployed and socially isolated (Bellack et al., 2007). Social cognition - defined as the set of mental operations that underlie social interactions - has emerged in recent years as a key factor in explaining these functional impairments (Fett et al., 2011) both directly, and also by mediating the relationship between neurocognition and functional outcome (Vauth et al., 2004; Brekke et al., 2005; Addington et al., 2006; Green et al., 2008; McGlade et al., 2008; Schmidt et al., 2011). However, whether the current tools for assessing functional impairments in schizophrenia in clinical practice are sensitive enough to measure change in social cognition remains unclear.

The Global Assessment of Functioning (GAF) scale is the measure recommended by the Diagnostic and Statistical Manual of Mental Disorders (revised 4th ed., text rev.; DSM-IV-TR; American Psychiatric Association, 2000) for assessing social, occupational and psychological

\footnotetext{
Corresponding author at: Neuropsychiatric Genetics Research Group, Department of Psychiatry, St. James's Hospital, Dublin 8, Ireland. Tel.: +3531 8962467.

E-mail addresses: donoghug@tcd.ie,droberts@tcd.ie (G. Donohoe).
}

functioning. It is a subjective measure based on a clinician's opinion of a patient's level of functioning and has been found to be a reliable and valid tool (Jones et al., 1995). As a subjective measure however, it is highly influenced by the outward presentation of positive and negative symptoms, bizarre behaviours (Startup et al., 2002) and the verbal skills of the patient (Tso et al., 2010). It is unclear if the GAF scale is a reliable tool for assessing the functional difficulties associated with more subtle impairments in social cognition, once these typical positive and negative symptoms have been controlled for. Perhaps consequently, few social cognition studies have employed the GAF scale as an outcome measure (Fett et al., 2011). Furthermore, among the few studies that have used the GAF and found that social cognition is a mediator in the relationship between neurocognition and functioning, most did not account for variation in symptom severity (McGlade et al., 2008; Gard et al., 2009; Schmidt et al., 2011).

The aim of this study was to test the ability of the GAF scale to explain variance in functioning caused by social and neurocognitive impairments after symptom severity has been controlled for. As social cognition encompasses a broad range of different domains we chose to focus solely on theory of mind for two reasons: 1) Fett et al.'s (2011) meta-analysis reported that theory of mind was the social cognitive domain that 
explained the largest amount of variance in functioning and 2) a previous study from our group demonstrated that theory of mind mediated the relationship between basic neuropsychological function and functional outcome using another measure of functional outcome, the Independent Living Scale (Revheim and Medalia, 2004; McGlade et al., 2008). We attempted to replicate this previous finding using the GAF scale as the outcome measure of global function.

\section{Materials and methods}

Informed consent was obtained from 216 patients with a diagnosis of schizophrenia or schizoaffective disorder which we then confirmed using the Structured Clinical Interview for DSM-IV disorders. These patients were recruited from 5 sites across Ireland as part of the Resource for Psychosis Genomics in Ireland initiative (RPGI) and represented a subset of patients $(n=365)$ for whom full data on global assessment of functioning, neuropsychological performance, and social cognition measures were available. Inclusion criteria required that participants were aged 18 to 65 years, had no history of comorbid psychiatric disorder, had no substance abuse in the preceding 6 months, had no prior head injury with loss of consciousness, and had no history of seizures.

Clinical data ascertained included symptom severity and medication dosage. Measurement of symptom severity was based on 60 items relating to clinical signs and symptoms from the Operational Criteria Checklist for Psychotic Illness (OPCRIT) (McGuffin et al., 1991). Medication dosage was measured in terms of chlorpromazine equivalents. Global functioning was measured using the Global Assessment of Functioning Scale (APA, DSM-IV TR, 2000), a 0-100 clinical rated scale described as providing a global index of psychological, social and occupational functioning.

\subsection{Assessment of neuropsychological function and social cognition}

Social cognition was measured using two theory of mind tasks: (a) the 'Reading the Mind in the Eyes' test (Baron-Cohen et al., 2001) is a mental state decoding task in which subjects rate 36 photographs of eyes portraying different expressions and correctly identify the thought or feeling as one of the four descriptors printed around each photograph; (b) the 'Hinting task' (Corcoran et al., 1995) is a mental state reasoning task in which participants listen to 10 vignettes each describing a social interaction between two characters and infer the intent behind the hint after each vignette. Neuropsychological performance was assessed using a battery of tests selected to index the specific domains of impairment that commonly occur in schizophrenia, namely IQ episodic memory, working memory and sustained attention as previously described (Donohoe et al., 2009).

Analysis of symptom severity scores was based on a principal components analysis with varimax rotation of OPCRIT scores, yielding a fivefactor solution of positive, negative, disorganised, manic, and depressive symptoms, as typically described and as previously reported for this sample (Cummings et al., 2013). Furthermore, as all neuropsychological measures were significantly associated with the GAF, and in order to simplify further analyses, we created a composite score of neurocognition using extracted regression values from an un-rotated principal components analysis. A series of linear regression analyses was then used to assess the degree of variance in global function explained by symptom severity, medication, neurocognition and social cognition. All analyses were conducted using Statistical Package for the Social Sciences (SPSS inc.) version 18.0 .

\section{Results}

Sample characteristics and demographics are described in Supplementary table 1 . In order to create a composite neurocognition score an unrotated principal components analysis was conducted and regression scores for each measure extracted. Each neuropsychological measure was found suitable for data reduction as indicated by a significant $(p<.001)$ Bartlett's test while a Kaiser-Meyer-Olkin score of .85 indicated that our sample size was adequate for factor analysis. All six measures loaded significantly onto the first factor accounting for $50.74 \%$ of the variance. Based on the factor loadings, a regression factor score was computed for each participant (see Supplementary table 2). The resulting scale was tested for internal consistency (Cronbach's $\alpha=.52$ ) and constitutes the overall measure of neurocognition.

The composite neurocognitive score was significantly positively associated with the Hinting task $(r=.43, p<.001)$, the Eyes task $(r=.50$, $p<.001)$, and the GAF scale $(r=.31, p<.001)$. Scores on the GAF scale were significantly positively associated with Hinting task performance $(r=.29, p<.001)$ but not with Eyes task performance $(r=.13, p=.19)$ and thus the Eyes task was excluded from further analysis. In order to determine how much variance in global function was measured by social and neurocognitive function we conducted a series of regression analysis using GAF scale scores as the dependent variable. Firstly, when symptom severity and anti-psychotic medication were entered, the model explained $27.7 \%$ of variance in global functioning $(F(6,209)=13.35, p<.001)$, with each of Manic $(t(216)=2.16$, $p<.05)$, disorganized $(t(216)=-6.03, p<.001)$, and negative symptoms $(t(216)=-3.50, p<.01)$ explaining variation in function, but not positive symptoms or medication dosage $(p>0.05)$. Secondly, when hinting task performance and the neurocognitive composite score were entered as independent variables these together explained $12 \%$ of the variance in GAF scale scores $(F(2,203)=13.73, p<.001)$, with both neurocognition $(t(203)=2.37, p<.01)$ and social cognition $(t(203)=3.20, p<.05)$ independently predicting GAF scale scores.

Finally, to test whether social cognition was a predictor of GAF scale scores independent of the effects of symptom severity we performed a further regression analysis with symptom severity entered on the first step, followed by neurocognitive performance and hinting task performance on the second step and GAF scores again entered as the dependent variable. After the $25.2 \%$ of variation in GAF scores explained by symptom severity $\left(F_{\text {change }}(3,188)=21.14, p<.001\right)$, neurocognitive performance explained an additional $4.7 \%$ of variation $\left(F_{\text {change }}(1,187)=12.63\right.$, $p<.001$ ) while the Hinting task failed to explained any further variance $\left(r_{\text {change }}^{2}=.006, F_{\text {change }}(1,186)=1.63, p=.20\right)$.

\section{Discussion}

This study aimed to investigate the sensitivity of the GAF scale - the most widely used clinical assessment of function in schizophrenia - to variation in social cognitive ability. Social cognition has repeatedly been shown to predict real life social and occupational function, and indeed to mediate the effects of general cognitive function on outcome (Vauth et al., 2004; Brekke et al., 2005; Addington et al., 2006; Green et al., 2008; McGlade et al., 2008; Schmidt et al., 2011). Despite this, our study demonstrates that GAF scores are largely insensitive to variation in social cognition. Of the $\sim 30 \%$ of variation explained by the variables considered here, $25 \%$ was explained by variation in symptom severity, only $~ 5 \%$ was explained by neuropsychological function, and performance on social cognition failed to explain any further variance in GAF scores. These findings are consisted with a recent study in 87 patients with bipolar disorder (Martino et al., 2011).

Despite the GAF being the most widely used measure in clinical settings (as recommended by DSM-IV-TR), most researchers have tended to use measurements other than the GAF scale to assess functioning (Fett et al., 2011). We believe that the results of our study help explain researchers' bias towards avoiding the GAF. In one study that did report a relationship between GAF scores and social cognition, the authors did not account for variance in GAF scores explained by symptom severity in their model (Schmidt et al., 2011). By contrast, our study suggest that when symptom severity has been accounted for, social cognition explains little of the variance in GAF scores. This is in contrast to other measures of social and functional outcome. For example, Tso et al. 
(2010) found that verbal memory was the only significant predictor of the GAF whereas all neurocognitive and social cognitive variables assessed predicted Social Adjustment Scale-Self-Report Scores (Weissman and MHS Staff, 1999). They argued that this difference reflected the subjective nature of the GAF scale as it would be heavily influenced by the verbal abilities of the patients rather than by a true assessment of their psychosocial functioning abilities. Our finding that neurocognition but not social cognition predicted the GAF scale is supportive of these earlier results.

Limitations of our study include that only one social cognitive domain, theory of mind, was available for analysis in our study. Although this was the domain most highly associated with functional outcomes in previous studies (see Fett et al. (2011)), it is possible that the GAF scale may have been more sensitive to social cognition deficits in emotion perception/ recognition or social knowledge, as Brekke et al. (2005) suggest. Secondly, our results are cross-sectional and as such do not imply causality. It would be necessary to repeat this study after a follow-up period in order to determine whether change in social cognitive domains might better explain variance in the GAF scale compared to a measurement taken at one time only. Finally, we excluded participants who had a history of substance misuse within the previous 6 months. Although this was to control for the effects of drug and alcohol use on the measured variables, substance misuse commonly accompanies schizophrenia (Turkington et al., 2009) and as such our sample may not be representative of all individuals with schizophrenia.

In conclusion, although the GAF scale is reported to be a valid measure of function and, as such, a useful tool for clinicians to assess patients' progress in rehabilitation, it is a less sensitive tool for establishing and monitoring improvements in function associated with social aspects of cognition, at least as measured by theory of mind. As a result of the recognition that social cognition is important to real world outcomes in schizophrenia, the number of social cognitive remediation trials is increasing (Kurtz and Richardson, 2012). It is likely that as our understanding of social cognition increases and as remediation program become more frequently incorporated into treatment plans for schizophrenia, a new scale will be needed for clinical use which can more sensitively monitor progress in these domains than the largely symptomatically based GAF scale.

\section{Role of funding source}

No funding body had any part in how this study was conducted or in how this manuscript was written.

\section{Contributors}

GD and DR designed the study. DR, GD, AH, EK, DM, MG, AC carried out the statistical analyses. All authors contributed to the writing and editing of the final manuscript and have approved its contents.

\section{Conflict of interest}

All authors disclose that they have no conflict of interest in relation to the publication of this manuscript and its contents.

\section{Acknowledgments}

We thanks all patient and staff who participated in the collection of patient data Thanks Prof Ted Dinan, Prof Kieran Murphy, Prof John Waddington, Prof Colm McDonald, Prof Eadbhard O'Callaghan, and Dr. Anthony O'Neil for their participation in recruitment of samples. Recruitment of the patient sample was supported by funding awards from the Wellcome Trust and Science Foundation Ireland (SFI) to AC \& MG and a Health Research Board (Ireland) grant to GD.

\section{Appendix A. Supplementary data}

Supplementary data to this article can be found online at http://dx.doi. org/10.1016/j.schres.2013.01.016.

\section{References}

Addington, J., Saeedi, H., Addington, D., 2006. Facial affect recognition: a mediator between cognitive and social functioning in psychosis? Schizophr. Res. 85 (1-3), 142-150.

American Psychiatric Association, 2000. Diagnostic and Statistical Manual of Mental Disorders. Author, Washington, DC

Baron-Cohen, S., Wheelwright, S., Hill, J., Raste, Y., Plumb, I., 2001. The "Reading the Mind in the Eyes" test revised version: a study with normal adults, and adults with Asperger syndrome or high-functioning autism. J. Child Psychol. Psychiatry 42 (2), 241-251.

Bellack, A.S., Green, M.F., Cook, J.A., Fenton, W., Harvey, P.D., Heaton, R.K., Laughren, T., Leon, A.C., Mayo, D.J., Patrick, D.L., Patterson, T.L., Rose, A., Stover, E., Wykes, T., 2007. Assessment of community functioning in people with schizophrenia and other severe mental illnesses: a white paper based on an NIMH-sponsored workshop. Schizophr. Bull. 33 (3), 805-822.

Brekke, J., Kay, D.D., Lee, K.S., Green, M.F., 2005. Biosocial pathways to functional outcome in schizophrenia. Schizophr. Res. 80 (2-3), 213-225.

Corcoran, R., Mercer, G., Frith, C.D., 1995. Schizophrenia, symptomatology and social inference: investigating "theory of mind" in people with schizophrenia. Schizophr. Res. 17 (1), 5-13.

Cummings, E., Donohoe, G., Hargreaves, A., Moore, S., Fahey, C., Dinan, T.G., McDonald, C., O'Callaghan, E., O'Neill, F.A., Waddington, J.L., Murphy, K.C., Morris, D.W., Gill, M., Corvin, A., 2013. Mood congruent psychotic symptoms and specific cognitive deficits in carriers of the novel schizophrenia risk variant at MIR-137. Neurosci. Lett. 532, $33-38$.

Donohoe, G., Walters, J., Morris, D.W., Quinn, E.M., Judge, R., Norton, N., Giegling, I., Hartmann, A.M., Möller, H.J., Muglia, P., Williams, H., Moskvina, V., Peel, R., O'Donoghue, T., Owen, M.J. O'Donovan, M.C., Gill, M., Rujescu, D., Corvin, A. 2009. Influence of NOS1 on verbal intelligence and working memory in both patients with schizophrenia and healthy control subjects. Arch. Gen. Psychiatry 66 (10), 1045-1054.

Fett, A.K., Viechtbauer, W., Dominguez, M.D., Penn, D.L., van Os, J., Krabbendam, L., 2011. The relationship between neurocognition and social cognition with functional outcomes in schizophrenia: a meta-analysis. Neurosci. Biobehav. Rev. 35 (3), 573-588.

Gard, D.E., Fisher, M., Garrett, C., Genevsky, A., Vinogradov, S., 2009. Motivation and its relationship to neurocognition, social cognition, and functional outcome in schizophrenia. Schizophr. Res. 115 (1), 74-81.

Green, M.F., Penn, D.L., Bentall, R., Carpenter, W.T., Gaebel, W., Gur, R.C., Kring, A.M., Park, S., Silverstein, S.M., Heinssen, R., 2008. Social cognition in schizophrenia: an NIMH workshop on definitions, assessment, and research opportunities. Schizophr. Bull. 34 (6), $1211-1220$.

Jones, S.H., Thornicroft, G., Coffey, M., Dunn, G., 1995. A brief mental health outcome scalereliability and validity of the Global Assessment of Functioning (GAF). Br. J. Psychiatry 166 (5), 654-659.

Kurtz, M.M., Richardson, C.L., 2012. Social cognitive training for schizophrenia: a metaanalytic investigation of controlled research. Schizophr. Bull. 38, 1092-1104.

Martino, D.J., Strejilevich, S.A., Fassi, G., Marengo, E., Igoa, A., 2011. Theory of mind and facial emotion recognition in euthymic bipolar I and bipolar II disorders. Psychiatry Res. 189 (3), 379-384.

McGlade, N., Behan, C., Hayden, J., O'Donoghue, T., Peel, R., Haq, F., Gill, M., Corvin, A., O'Callaghan, E., Donohoe, G., 2008. Mental state decoding v. Mental state reasoning as a mediator between cognitive and social function in psychosis. Br. J. Psychiatry 193 (1), 77-78

McGuffin, P., Farmer, A., Harvey, I., 1991. A polydiagnostic application of operational criteria in studies of psychotic. Arch. Gen. Psychiatry 48 (8), 764-770.

Revheim, N., Medalia, A., 2004. The independent living scales as a measure of functional outcome for schizophrenia. Psychiatr. Serv. 55 (9), 1052-1054.

Schmidt, S.J., Mueller, D.R., Roder, V., 2011. Social cognition as a mediator variable between neurocognition and functional outcome in schizophrenia: empirical review and new results by structural equation modeling. Schizophr. Bull. 37 (Suppl. 2), S41-S54.

Startup, M., Jackson, M.C., Bendix, S., 2002. The concurrent validity of the Global Assessment of Functioning (GAF). Br. J. Clin. Psychol. 41 (Pt 4), 417-422.

Tso, I.F., Grove, T.B., Taylor, S.F., 2010. Emotional experience predicts social adjustment independent of neurocognition and social cognition in schizophrenia. Schizophr. Res. 122 (1-3), 156-163.

Turkington, A., Mulholland, C.C., Rushe, T.M., Anderson, R., McCaul, R., Barrett, S.L., Barr, R.S., Cooper, S.J., 2009. Impact of persistent substance misuse on 1-year outcome in firstepisode psychosis. Br. J. Psychiatry 195 (3), 242-248.

Vauth, R., Rusch, N., Wirtz, M., Corrigan, P.W., 2004. Does social cognition influence the relation between neurocognitive deficits and vocational functioning in schizophrenia? Psychiatry Res. 128 (2), 155-165.

Weissman, M.H.S. Staff, 1999. Social Adjustment Scale-Self-Report (SAS). Multi-Health Systems Inc., Tonawanda, NY. 